\title{
Mitochondrial membrane potential regulates matrix configuration and cytochrome $c$ release during apoptosis
}

\author{
E Gottlieb $^{1,2}$, SM Armour ${ }^{1,2}$, MH Harris $^{1,2}$ and CB Thompson ${ }^{\star, 1,2}$ \\ 1 Abramson Family Cancer Research Institute, University of Pennsylvania, \\ Philadelphia, PA 19104, USA \\ 2 Department of Cancer Biology, University of Pennsylvania, Philadelphia, PA \\ 19104, USA \\ * Corresponding author: CB Thompson, University of Pennsylvania, Abramson \\ Family Cancer Research Institute, 421 Curie Blvd., Room 450 BRB II/III, \\ Philadelphia, PA 19104-6160, USA. Tel: 215-746-5515; Fax: 215-746-5511; \\ E-mail: craig@mail.med.upenn.edu
}

Received 23.9.02; revised 5.1.03; accepted 31.1.03 Edited by M Peter

\begin{abstract}
During apoptosis, the mitochondrial membrane potential (MMP) decreases, but it is not known how this relates to the apoptotic process. It was recently suggested that cytochrome $c$ is compartmentalized in closed cristal regions and therefore, matrix remodeling is required to attain complete cytochrome $c$ release from the mitochondria. In this work we show that, at the onset of apoptosis, changes in MMP control matrix remodeling prior to cytochrome $c$ release. Early after growth factor withdrawal the MMP declines and the matrix condenses. Both phenomena are reversed by adding oxidizable substrates. In mitochondria isolated from healthy cells, matrix condensation can be induced by either denying oxidizable substrates or by protonophores that dissipate the membrane potential. Matrix remodeling to the condensed state results in cristal unfolding and exposes cytochrome $c$ to the intermembrane space facilitating its release from the mitochondria during apoptosis. In contrast, when a transmembrane potential is generated due to either electron transport or a $\mathrm{pH}$ gradient formed by acidifying the medium, mitochondria maintain an orthodox configuration in which most cytochrome $c$ is sequestered in the cristae and is resistant to release by agents that disrupt the mitochondrial outer membrane.

Cell Death and Differentiation (2003) 10, 709-717. doi:10.1038/ sj.cdd. 4401231
\end{abstract}

Keywords: apoptosis; mitochondrial membrane potential; matrix configuration; cytochrome $c$

Abbreviations: MMP, mitochondrial membrane potential; EM, electron microscopy; TMRE, tetramethyl-rhodamine ethyl ester; CCCP, carbonylcyanide $m$-chlorophenylhydrazone; $\mathrm{BH} 3, \mathrm{Bcl}-2$ homology region-3; MIB, mitochondrial isolation buffer; MRB, mitochondrial reaction buffer.

\section{Introduction}

In addition to being the source of energy that supports life under aerobic conditions, mitochondria can also be the source of signals that initiate apoptotic cell death. ${ }^{1}$ Mitochondria contain key regulators of caspases; a family of proteases that are major factors in many apoptotic processes. ${ }^{2}$ The release of cytochrome $c$ from the mitochondrial intermembrane space induces the assembly of the apoptosome that is required for activating downstream caspases. ${ }^{3,4}$ Cytochrome $c$ release from mitochondria is a key event in initiating apoptosis, but the actual mechanism of its release is still debatable. In particular, the relation between mitochondrial physiology and the release of cytochrome $c$ and other apoptogenic factors from mitochondria is not clear.

Mitochondria utilize oxidizable substrates to produce a membrane potential in the form of a proton gradient across the mitochondrial inner membrane. It was shown recently that the supply of oxidizable substrates to mitochondria depends on the concentration of external growth factors. ${ }^{5}$ Withdrawal of growth factors or loss of the extracellular glucose supply will lead to a decline in mitochondrial membrane potential (MMP). If growth factor or glucose deprivation persists, cells ultimately undergo apoptosis that is initiated by cytochrome $c$ release from mitochondria. ${ }^{5,6}$ Whether changes in mitochondrial physiology contribute to the initiation of cell death in response to growth factor withdrawal remains a controversial issue. ${ }^{7}$

It was recently suggested that structural changes in mitochondria may be required to achieve complete and rapid cytochrome $c$ release. ${ }^{8}$ In this work, we demonstrate a direct relation between mitochondrial structure and function. A decrease in MMP leads to matrix condensation and exposure of cytochrome $c$ to the intermembrane space, facilitating cytochrome $c$ release and cell death following an apoptotic insult.

\section{Results}

\section{Mitochondrial matrix condenses because of substrate limitation after IL-3 deprivation}

We used the IL-3-dependent FL5.12 cell line to examine mitochondrial structure following growth factor withdrawal. Electron microscopy (EM) analysis was performed at 6 and $12 \mathrm{~h}$ after IL-3 withdrawal and compared to electron micrographs of cells maintained with IL-3. It is important to note that the cells were still viable at both time points of analysis and more than $90 \%$ of the cells were capable of recovery, as assayed by clonogenicity in IL-3-containing medium (data not shown). Changes in mitochondrial morphology 6 and $12 \mathrm{~h}$ after IL-3 removal were observed by EM (Figure 1a). The mitochondria became progressively smaller than those in 
a

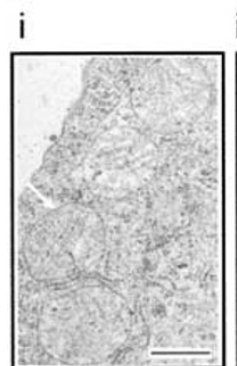

$+\mathrm{IL}-3$ ii

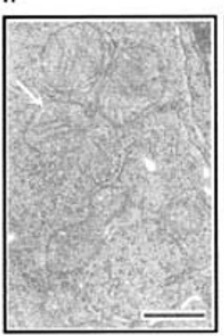

-IL-3; 6 hours iii

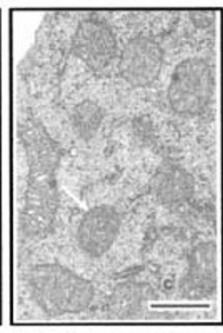

-IL-3; 12 hours

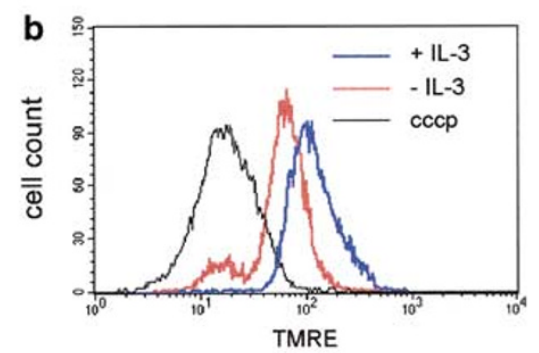

c

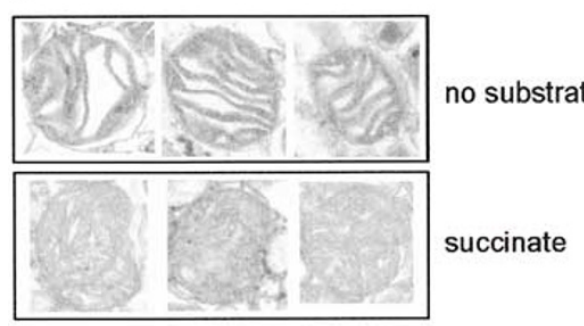

d

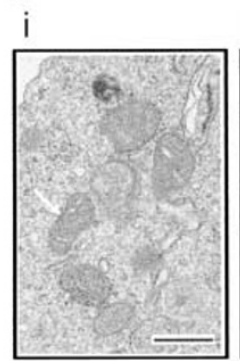

-IL- $3 ; 12$ hours ii

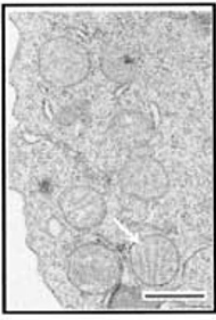

$-\mathrm{IL}-3$

+ mSucc

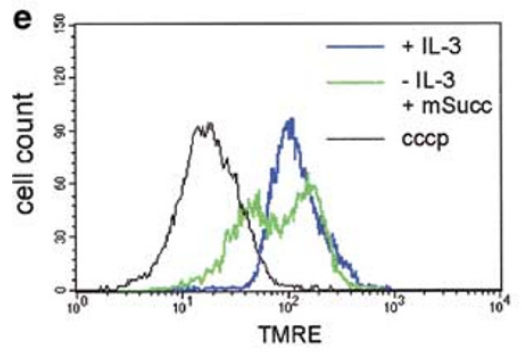

Figure 1 Mitochondrial matrix condenses because of the limitation in oxidizable substrates after IL-3 withdrawal. (a) FL5.12 cells were grown in the presence (i) or absence of IL-3 for $6 \mathrm{~h}$ (ii) or $12 \mathrm{~h}$ (iii) and fixed for EM analysis. The bar represents $500 \mathrm{~nm}$ length and the white arrows point at one representative mitochondrion in each panel. (b) Cells were grown in the presence or absence of IL-3 for $12 \mathrm{~h}$, followed by MMP analysis with $25 \mathrm{nM}$ TMRE staining followed by FACS analysis. The protonophore CCCP was used to dissipate the membrane potential and to define the baseline for the analysis of mitochondrial potential by TMRE fluorescence. $50 \mu \mathrm{M}$ CCCP was added to cells growing in the presence of IL-3 5 min before the FACS analysis. (c) Mitochondria were isolated from cells growing with IL-3 and incubated in MRB with or without $7 \mathrm{mM}$ succinate for $10 \mathrm{~min}$ before fixation and EM analysis. (d) Cells were incubated without IL-3 for $12 \mathrm{~h}$ (i, ii). Where indicated (ii), $5 \mathrm{mM}$ methyl succinate (mSucc) was added to cells growing in the absence of IL-3 $15 \mathrm{~min}$ before fixation and analysis. (e) A measure of $5 \mathrm{mM}$ methyl succinate was added every $3 \mathrm{~h}$ to cells growing in the absence of IL-3. TMRE staining followed by FACS analysis was used to assess MMP. A comparative analysis of untreated (+IL-3) and treated (-IL-3 $+m S u c c$ ) cells is presented. (f) Viability analysis of cells incubated in the absence of IL-3 either with or without methyl succinate (as in panel E). Viability was determined by propidium iodide exclusion and assessed by FACS analysis

control cells, and the matrix became increasingly more dense. This condensation correlates with a decrease in MMP, as assessed by staining with the lipophilic cationic dye tetramethyl-rhodamine ethyl ester (TMRE) (Figure 1b). In subsequent experiments, $12 \mathrm{~h}$ of IL-3 withdrawal was used because a reproducible decline in MMP is observed (Figure 1b). In contrast, at later time points, when cells are initiating apoptosis, paradoxical increases in MMP have been reported, ${ }^{9-11}$ which may represent dequenching of rhodaminebased dyes or the metabolic acidosis that occurs under conditions of reduced ATP synthesis.

When mitochondria, isolated from FL5.12 cells growing in the presence of IL-3, were incubated in vitro in a buffer lacking oxidizable substrates, they assumed a condensed configura- tion (Figure 1c). Addition of succinate, an electron donor to complex II of the respiratory chain (succinate dehydrogenase), resulted in matrix expansion to the 'orthodox configuration' normally observed in healthy cells (Figure 1c), demonstrating that the availability of oxidizable substrates plays a role in regulating matrix configuration. ${ }^{12}$ Therefore, it was plausible that in growth factor-deprived cells, substrate deficiency was the reason for matrix condensation. To address this possibility, cells were withdrawn from IL-3 for $12 \mathrm{~h}$ and then incubated for $15 \mathrm{~min}$ in the presence of methyl succinate, a membrane permeable substrate of complex II. EM analysis showed that mitochondria responded to methyl succinate by readopting an orthodox configuration (Figure 1d), indicating that matrix condensation following IL-3 with- 
drawal is the result of substrate deficiency. In addition, the decrease in MMP following IL-3 withdrawal was partially prevented by methyl succinate (Figure 1e). To evaluate the importance of substrate-regulated mitochondrial configuration to apoptosis, FL5.12 cells were maintained in the absence of IL-3 with or without periodic additions of $5 \mathrm{mM}$ methyl succinate every $3 \mathrm{~h}$. Viability analysis demonstrated significant protection from apoptosis by methyl succinate (Figure 1f).

\section{MMP regulates matrix configuration}

The above data suggested that matrix condensation in IL-3deprived cells is because of a decline in oxidizable substrates and a consequent drop in MMP. To test if mitochondrial structure is regulated by membrane potential, we pharmacologically manipulated the MMP of FL5.12 cells that were maintained in the presence of IL-3 and followed changes in mitochondrial structure by EM analysis. Rotenone blocks respiration at complex I of the mitochondrial respiratory chain (NADH dehydrogenase) and therefore decreases the redoxdependent proton pumping by complexes I, III and IV. Incubating cells with rotenone for $15 \mathrm{~min}$ resulted in a decline in MMP (Figure 2ai) that was comparable to that of IL-3deprived cells (Figure 1b). EM analysis showed that mitochondria became condensed in response to rotenone treatment even in the presence of IL-3 (Figure 2aii and iii). Oligomycin is a blocker of ATP synthase that prevents proton passage through the enzyme, thus inducing mitochondrial membrane hyperpolarization. Treatment of cells that were maintained in the presence of IL-3 with oligomycin for 5 min resulted in mitochondrial swelling beyond the orthodox configuration (Figure 2bii). To confirm that mitochondrial swelling was because of hyperpolarization, the protonophore carbonylcyanide $m$-chlorophenylhydrazone (CCCP) was added to depolarize the mitochondria in oligomycin-treated cells (Figure 2bi). Bypassing ATP synthase with CCCP resulted in reversal of the mitochondrial swelling (Figure 2biii). However, the mitochondria of oligomycintreated cells do not condense as completely with CCCP as the mitochondria from cells treated with rotenone. This appears to result from the associated depolarization of the plasma membrane in intact cells, because isolated mitochondria treated with CCCP in the presence of substrate undergo condensation equivalent to that achieved with rotenone treatment (Figure 3 ).

To avoid cytosolic effects on mitochondria during treatment, and to explore the direct relations between MMP and mitochondrial configuration, we turned to an in vitro analysis of isolated mitochondria, in which membrane potential could be regulated by manipulating the availability of oxidizable substrates to the respiratory chain and by protonophores. Mitochondria were isolated from FL5.12 cells growing in the presence of $\mathrm{IL}-3$ and MMP was analyzed in vitro using the rhodamine 123 quenching technique. ${ }^{13}$ Matrix configuration was determined either by EM analysis, or by light scattering the more condensed the matrix, the more light the mitochondria diffract. ${ }^{13,14}$ When mitochondria were incubated in the absence of oxidizable substrates and in the presence of rotenone, light scattering and rhodamine 123 fluorescence were high, indicating matrix condensation and low MMP, respectively (Figure 3ai). Upon addition of succinate, an
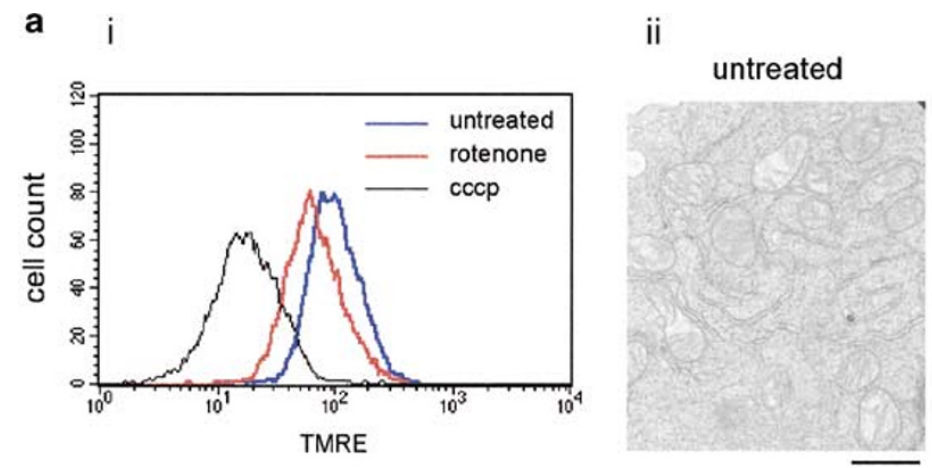

iii
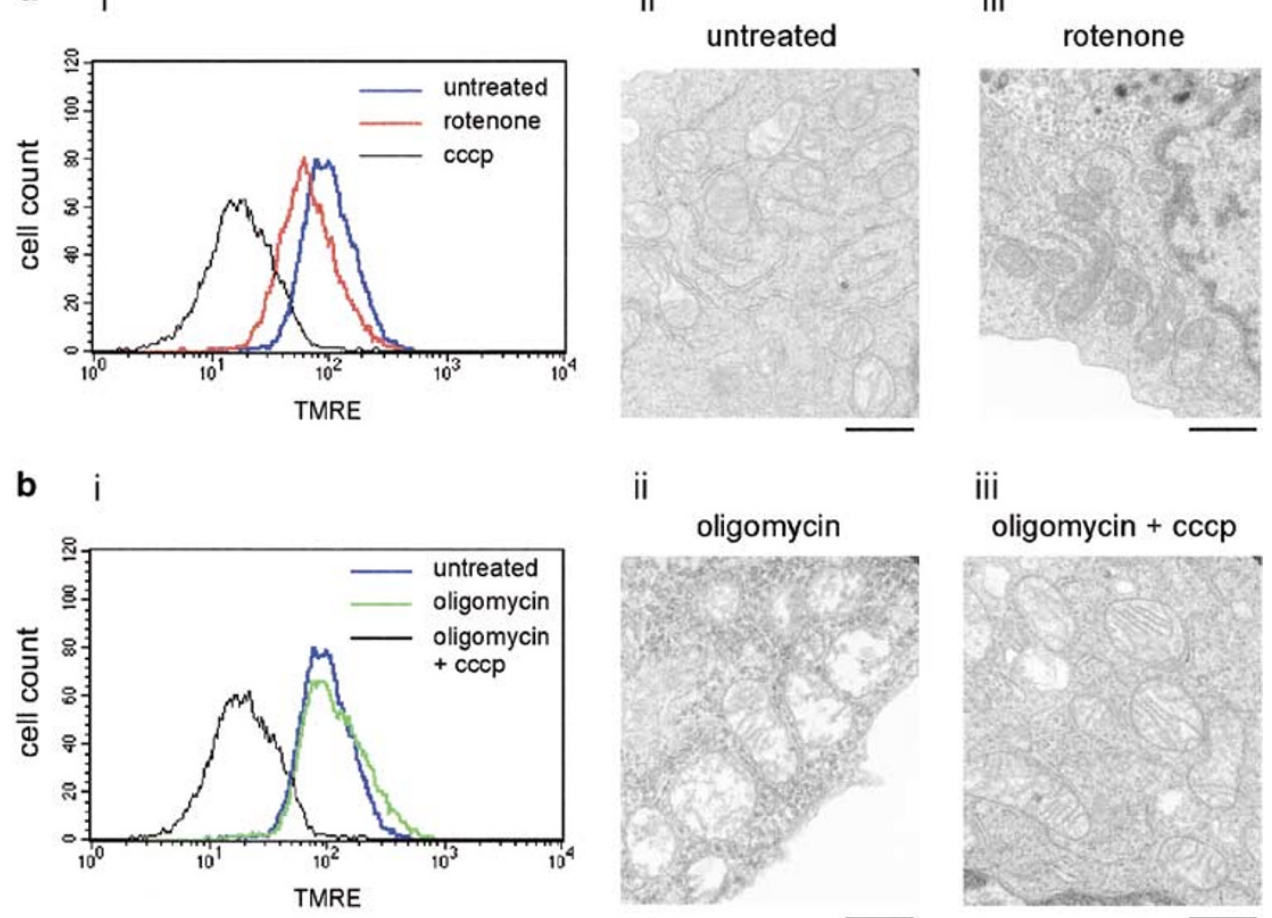

iii

oligomycin $+\operatorname{cccp}$

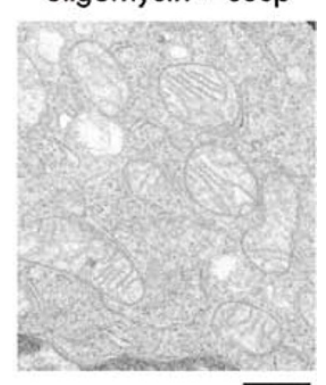

Figure 2 MMP regulates matrix configuration in cells. (a) Cells growing in the presence of IL-3 were either left untreated or treated with $5 \mu \mathrm{M}$ rotenone for 15 min prior to TMRE staining and FACS analysis (i) or EM (ii, iii) analysis as in Figure 1. (b) Cells growing in the presence of IL-3 were treated with $10 \mu \mathrm{g} / \mathrm{ml}$ oligomycin for 5 min, with or without $50 \mu \mathrm{M}$ CCCP and analyzed by FACS (i) or EM (ii, iii). The bar represents $500 \mathrm{~nm}$ length 


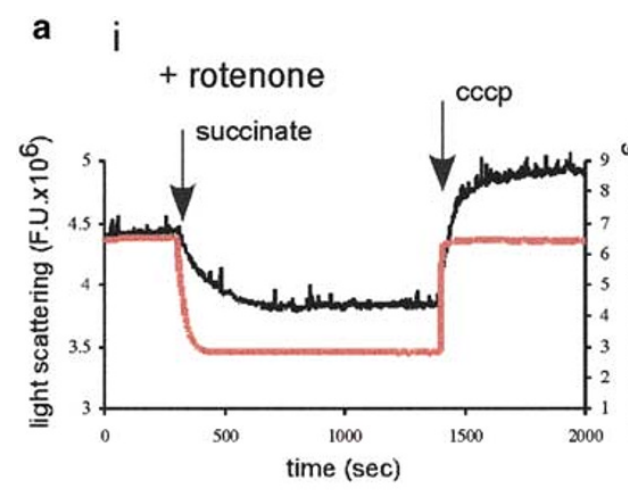

b i - rotenone
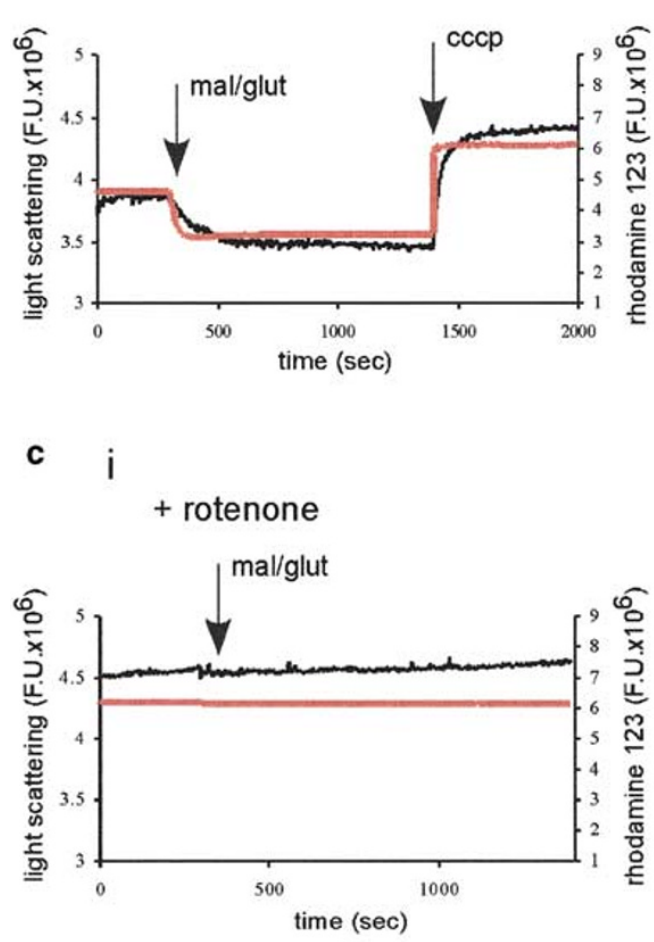

ii

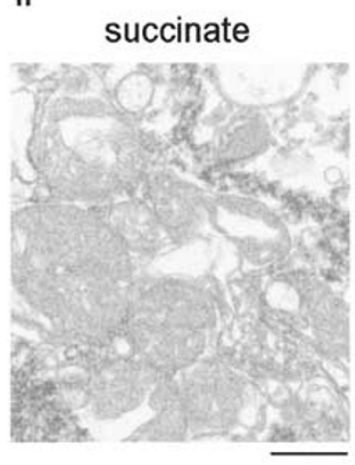

ii

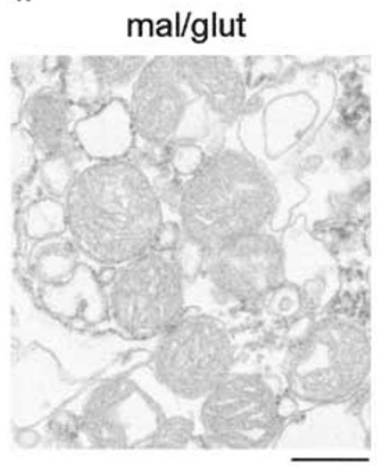

ii

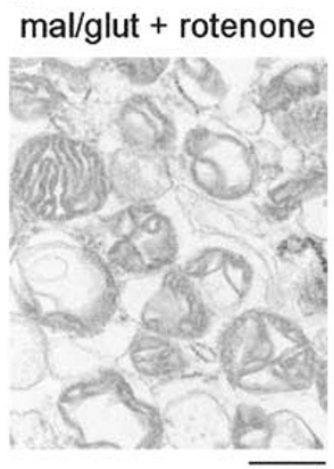

iii

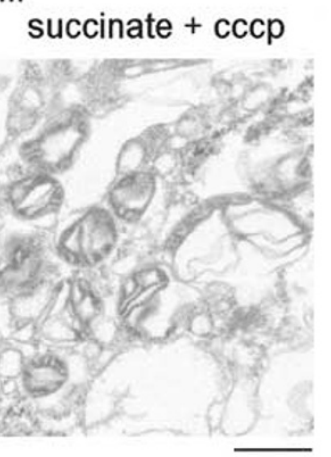

iii $\mathrm{mal} / \mathrm{glut}+\mathrm{cccp}$

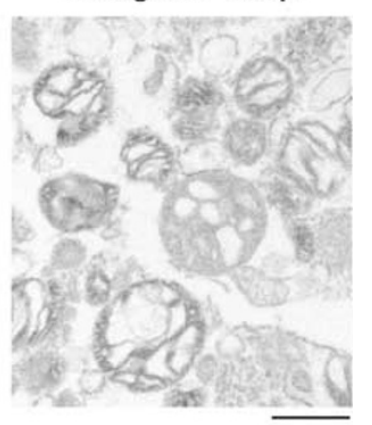

Figure 3 MMP regulates matrix configuration in vitro. (a-c) Mitochondria were isolated from FL5.12 cells growing in the presence of IL-3 and analyzed for membrane potential using rhodamine 123 quenching (red lines) and for matrix configuration using light scattering (black lines) in a spectrofluorometer (i) or by EM (ii and iii) under the indicated conditions. The bar represents $500 \mathrm{~nm}$ length; $\mathrm{FU}=$ fluorescent units; mal/glut=malate and glutamate

immediate increase in MMP was detected followed by a delayed change in light diffraction (Figure 3ai). This change in diffraction resulted from conversion of the matrix to the orthodox configuration as was confirmed by EM (Figure 3aii). Addition of CCCP to the assay resulted both in a dissipation of the membrane potential and in an increase in mitochondrial light diffraction (Figure 3ai). EM analysis confirmed that the matrix recondensed (Figure 3aiii). The same results were observed when mitochondria were treated with malate and glutamate that supply electrons to complex I of the respiratory chain by increasing mitochondrial NADH (Figure 3b). In this case, rotenone was omitted from the assay to enable complex I activity, therefore mitochondria maintained some of their membrane potential even without exogenous substrates, because of endogenous NADH (Figure 3bi). This partial potential could be dissipated with rotenone (compare the initial level of fluorescence in Figure 3bi to 3ai). When malate and glutamate were added to mitochondria, a rapid increase in MMP was detected and followed by a configuration change (Figure 3bi and ii). As was the case when succinate was used as an electron donor, adding CCCP resulted in a decline of the membrane potential and in matrix condensation (Figure 3bi and iii). In contrast, when malate and glutamate were added to mitochondria in the presence of rotenone, no change in membrane potential or matrix configuration was detected (Figure 3c). This indicates both that these mitochondrial 
modifications were not because of changes in ionic strength caused by the addition of substrates, and that matrix condensation can occur independently of the respiratory rate; since malate- and glutamate-energized mitochondria respire at their maximal rate with CCCP, and do not respire in the presence of rotenone (data not shown).

To verify that following mitochondrial depolarization the matrix becomes more condensed, a morphometric analysis was performed. In the EM analysis, increased opacity of the matrix resulted from increased diffraction of electrons, making the matrix appear darker in the image. Therefore, the more condensed the matrix, the more electrons it diffracts and the darker it appears on the micrograph. Using Scion Image for Windows (Scion Corporation), electron micrographs were converted to a density plot, in which the Z-axis is representative of the density of each pixel. Digital pixel density is numerically expressed from 0 to 255 , and a color gradient from white to red was then applied to better distinguish between areas of different densities. In a typical mitochondrion that was incubated in the presence of succinate (orthodox configuration), the matrix is less dense and occupies more area (in a 3D image - this will represent volume) than matrix from a mitochondrion incubated with succinate and CCCP (Figure 4ai and ii). A density threshold above 50 was used to define the matrix area and 25 mitochondria from each treatment were analyzed for matrix density and the percent of image area occupied by matrix. These results showed a significant increase in matrix density $(P<0.0001$; Student's $t$-test) and a decrease in matrix area $(P<0.0001)$ in depolarized mitochondria as compared to energized mitochondria, providing objective evidence that matrix volume decreases and density increases when mitochondria are depolarized (Figure $4 b$ and $c$ ).

The above results suggest a connection between MMP and mitochondrial matrix configuration. To confirm that the rhodamine 123 quenching assay measures changes in MMP and not mitochondrial volume, we examined the relation of mitochondrial volume to MMP in purified mitochondria in which the volume is manipulated by stepwise addition of sucrose to the medium. Mitochondria were initially incubated in $100 \mathrm{mM}$ sucrose buffer. Light scattering and rhodamine 123 fluorescence were analyzed during a i succinate

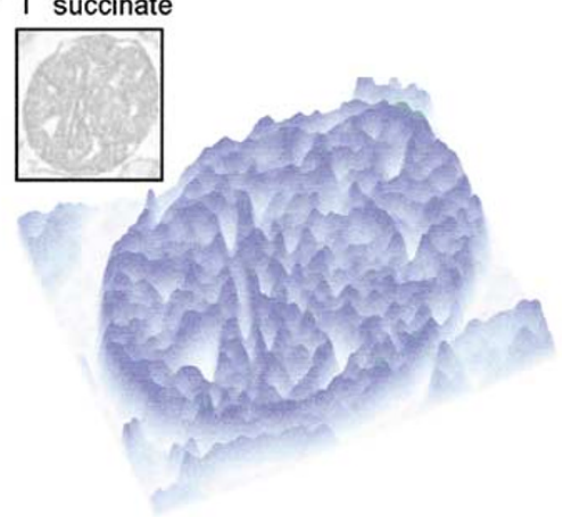

ii succinate + CCCP

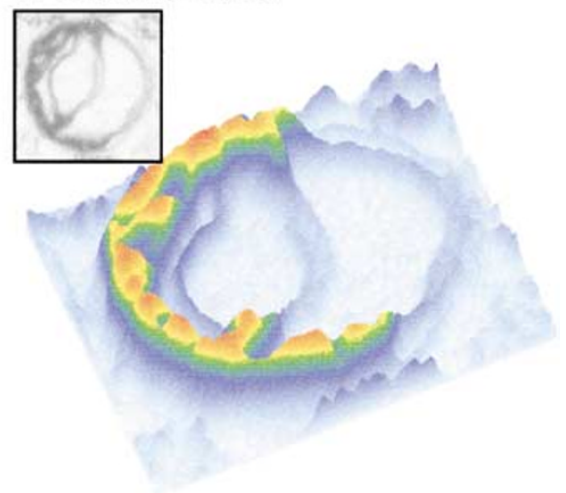

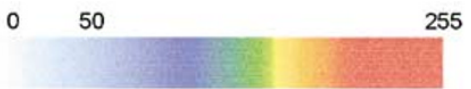
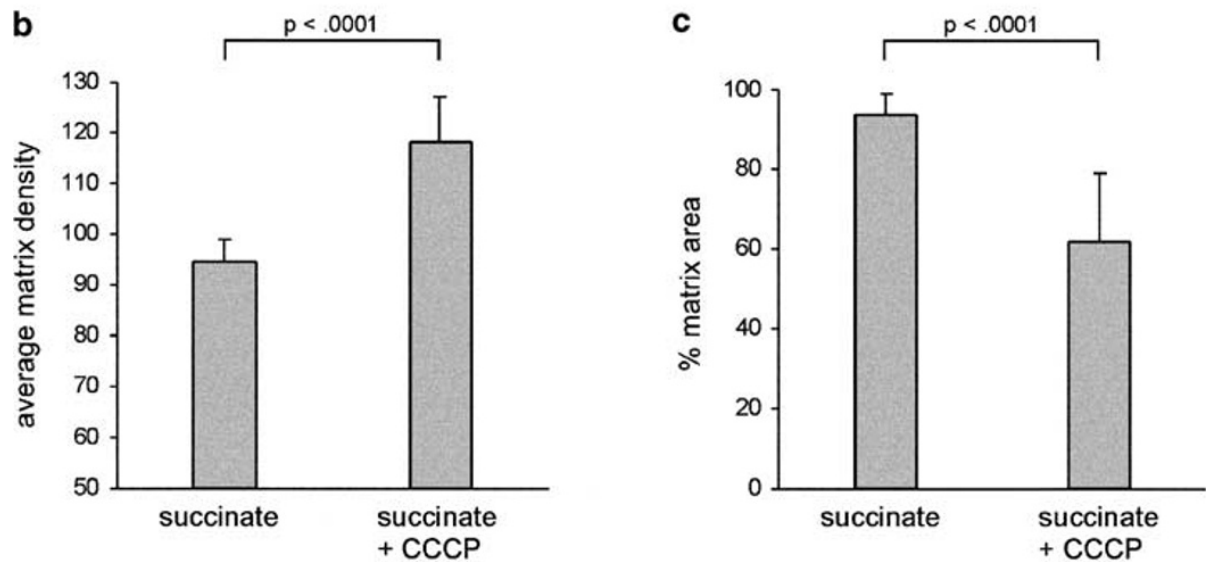

Figure 4 Depolarized mitochondria adopt a condensed configuration. (a) Mitochondria were incubated in MRB with $5 \mu \mathrm{M}$ rotenone and $7 \mathrm{mM}$ succinate with (ii) or without (i) $50 \mu \mathrm{M} \mathrm{CCCP} \mathrm{for} 10 \mathrm{~min}$. Following fixation and EM analysis, the digital images of individual mitochondria were analyzed by Scion Image for Windows to display color-mapped density on the Z-axis. Representative mitochondria of each treatment are presented. The matrix area was defined as a density unit above 50. (b and c) Based on the analysis described in panel A, 25 mitochondria from each treatment were analyzed for the average density of the matrix (b) or the percentage of the area that the matrix occupied in each image (c) 
consecutive increases in the concentration of sucrose (50 mM increments). While light scattering was significantly affected by the addition of sucrose (indicating a more condensed matrix), there was no apparent change in rhodamine 123 fluorescence (Figure 5a). This indicates that changes in matrix volume alone were not responsible for the changes in rhodamine 123 fluorescence we observed through manipulation of electron transport substrates and uncoupling agents. To confirm a direct relation between MMP and matrix configuration, mitochondria were incubated without oxidizable substrates in a slightly acidic environment $(\mathrm{pH}$ 6.4). By increasing the proton concentration outside the mitochondria, an artificial potential is created even when, because of lack of substrates, mitochondria are incapable of electron transport. EM analysis showed that substratedeprived, condensed mitochondria underwent decondensation of their matrix when incubated at $\mathrm{pH} 6.4$ for $10 \mathrm{~min}$, as compared to mitochondria at physiological pH (7.4) (Figure $5 b)$, demonstrating that the membrane potential can control mitochondrial configuration directly.
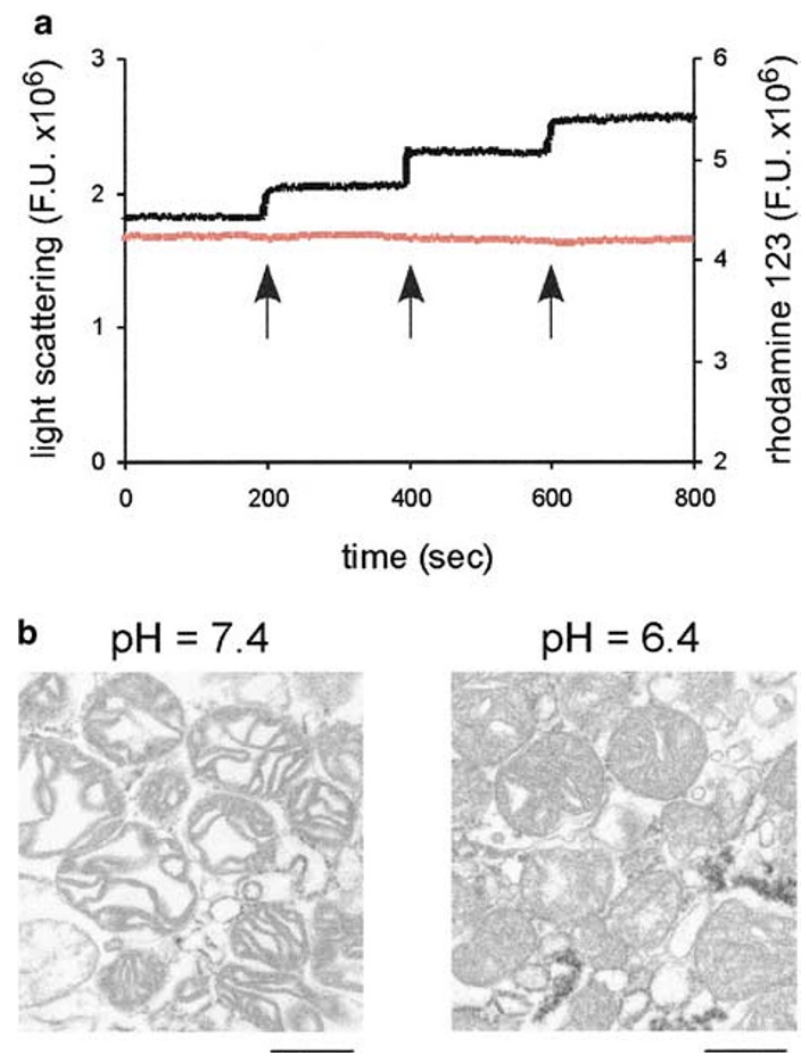

Figure 5 (a) Change in matrix volume but not in MMP does not affect rhodamine 123 quenching. Mitochondria were depolarized with $50 \mu \mathrm{M} \mathrm{CCCP}$ and $50 \mathrm{nM}$ valinomycin and incubated in $100 \mathrm{mM}$ sucrose buffer and light scattering (black line) or rhodamine 123 quenching analysis (red line) was performed as in Figure 3 . Sucrose levels were increased in $50 \mathrm{mM}$ increments every $200 \mathrm{~s}$ (arrows) to follow changes in matrix volume and rhodamine 123 fluorescence. (b) An artificial membrane potential in the absence of oxidizable substrates induces orthodox configuration. Isolated mitochondria were incubated without oxidizable substrates in MRB at normal $\mathrm{pH}(7.4)$ or at acidic $\mathrm{pH}(6.4)$ creating a respiration-independent proton gradient across the inner membrane. Following $10 \mathrm{~min}$ incubation, mitochondria were fixed and analyzed by EM. The bar represents $500 \mathrm{~nm}$ length

\section{Matrix condensation facilitates cytochrome $c$ release from mitochondria}

The fact that adding methyl succinate to IL-3-deprived cells resulted in mitochondrial matrix expansion and protection from apoptosis (Figure 1), suggested that mitochondrial structural changes may contribute to the subsequent step of initiating apoptosis through cytochrome $c$ release. It was suggested recently that most of the cytochrome $c$ in mitochondria is enclaved in the cristae. ${ }^{15}$ To test whether matrix condensation leads to unraveling of the cristae and contributes to exposure of cytochrome $c$ to the intermembrane space, we performed immuno-EM analysis using anticytochrome $c$ antibodies. When mitochondria were maintained in the orthodox configuration in the presence of succinate, cytochrome $c$ was compartmentalized in cristal regions (Figure 6a top panel). In contrast, when CCCP was added to mitochondria energized with succinate, to induce a condensed configuration, a higher percentage of cytochrome $c$ detected by immuno-gold staining was facing the expanded intermembrane space regions (Figure $6 a$ bottom panel). Since the matrix undergoes condensation, communication between intermembrane space and the intracristal space becomes more extensive. Owing to limitations in the fixation process of the samples for immuno-EM, these samples are not stained with osmium tetroxide to efficiently visualize matrix condensation. Therefore, in order to confirm that mitochondria adopted the orthodox or condensed configuration for each treatment, a parallel sample from each mitochondria prep was fixed and analyzed by standard EM analysis (data not shown). Moreover, it is still apparent from the immuno-EM analysis that when compared to succinate-energized mitochondria, depolarized mitochondria displayed larger nonmatrix areas (intermembrane space) as a result of matrix condensation (Figure 6a). To analyze the change of cytochrome $c$ distribution in mitochondria more than 50 mitochondria of each treatment were analyzed, and the percentage of detectable cytochrome $c$ in the immuno-EM that are oriented towards the intermembrane space was calculated. A threefold increase in cytochrome $c$ that is accessible to the intermembrane space was detected in mitochondria depolarized (condensed) with CCCP (Figure 6b).

To investigate the relation between matrix configuration and cytochrome $c$ release, we analyzed whether the condensed state of mitochondria, because of depolarization, could contribute to cytochrome $c$ release following treatment with a peptide from the $\mathrm{Bcl}-2$ homology region-3 $(\mathrm{BH} 3)$ of $\mathrm{Bid}, \mathrm{a}$ proapoptotic $\mathrm{Bcl}-2$ family member. Indeed, manipulations that induced matrix condensation significantly enhanced cytochrome $c$ release from mitochondria following the addition of $\mathrm{BH} 3$ peptides (Figure $6 \mathrm{c}$ ). This release was $\mathrm{BH} 3-$ dependent since a mutant $\mathrm{BH} 3$ peptide with two amino-acid substitutions did not cause release of cytochrome $c$ from mitochondria under any condition (Figure $6 \mathrm{c}$ ), indicating that matrix condensation alone does not induce cytochrome $c$ release. Accelerated $\mathrm{BH} 3$-induced cytochrome $c$ release was observed in mitochondria depolarized due to either a respiration block by rotenone in the presence of malate and glutamate, or to uncoupling of electron transport from the generation of a membrane potential by treatment with CCCP. These results 
a succinate
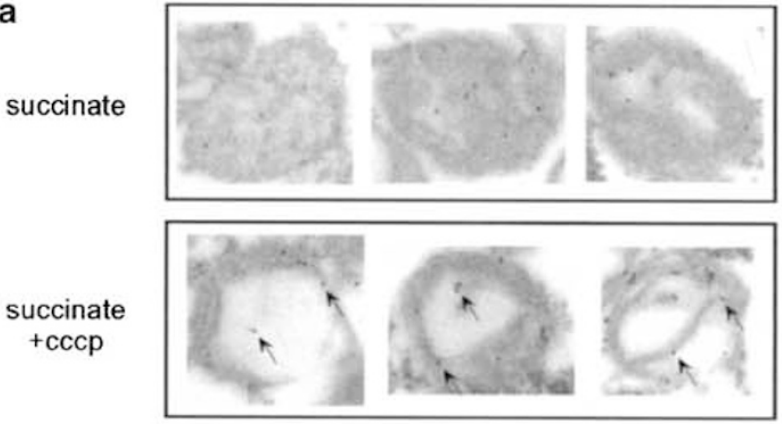

b

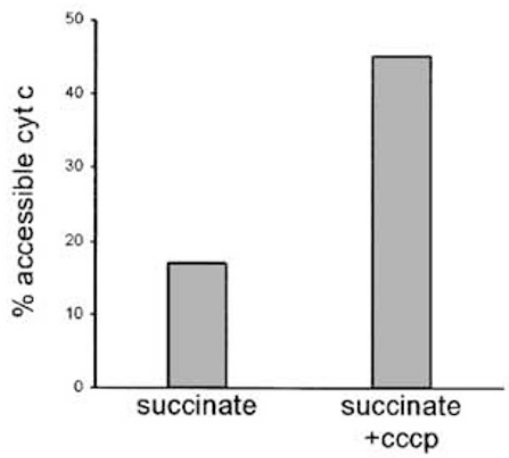

C

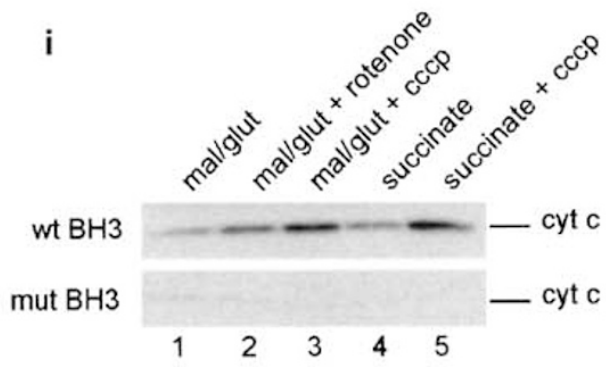

ii
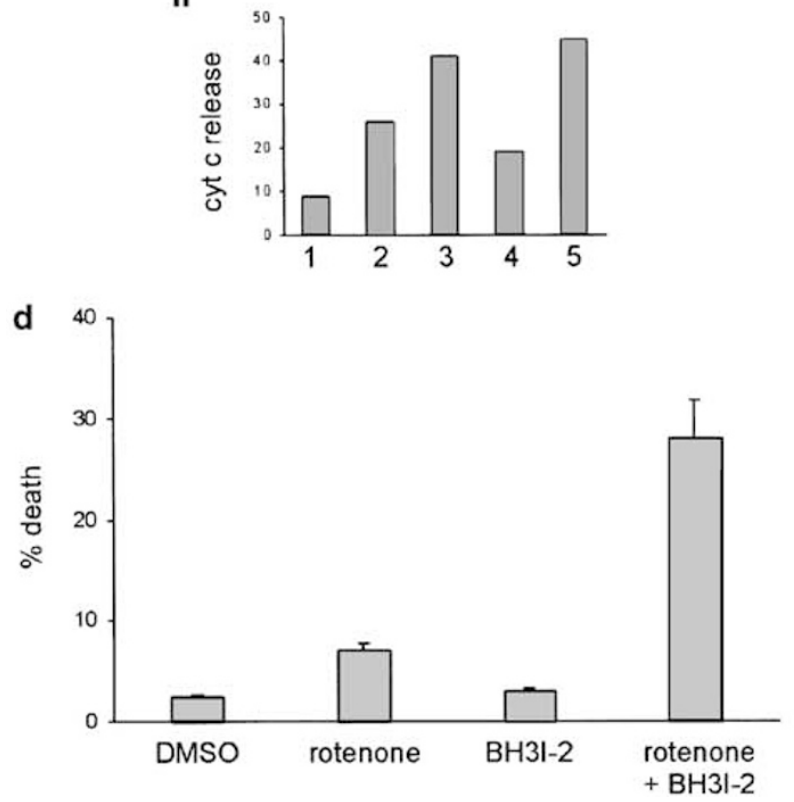

emphasize that cytochrome $c$ release is not affected by the rate of respiration, but rather by the potential.

Combined with the observation that in IL-3-deprived cells methyl succinate restored an orthodox configuration and delayed apoptosis, the in vitro results indicate that mitochondrial configuration, determined by changes in membrane potential, may play an important role in regulating cytochrome $c$ release and the susceptibility of cells to apoptosis. To test this possibility, cells were treated with rotenone to induce mitochondrial matrix condensation (Figure 2aiii) and viability was analyzed in the presence or absence of the cell-permeant $\mathrm{BH} 3-$ mimetic compound $\mathrm{BH} 3 \mathrm{I}-2 .{ }^{16}$ Cells that were incubated with $10 \mu \mathrm{M} \mathrm{BH} 3 \mathrm{I}-2$ or $5 \mu \mathrm{M}$ rotenone alone for $12 \mathrm{~h}$ showed very little signs of death (Figure $6 \mathrm{~d}$ ). On the other hand, a combined treatment with rotenone and $\mathrm{BH} 3 \mathrm{I}-2$ had a profound synergistic effect, and a significant acceleration of cell death was observed $12 \mathrm{~h}$ after treatment (Figure 6d).

\section{Discussion}

A decrease in MMP has been observed in response to many apoptotic stimuli, ${ }^{10,17,18}$ but the relation between an MMP decrease and the initiation of apoptosis remained unclear. It was recently shown that disruption of the mitochondrial outer membrane is not sufficient to achieve complete cytochrome $c$ release. $^{8,19}$ Studies of mitochondrial structure have suggested that most of the cytochrome $c$ protein is sequestered in the cristae ${ }^{15}$ and in order to achieve complete release of cytochrome $c$, as is generally observed during apoptosis, ${ }^{20}$ structural remodeling of the inner membrane is required. ${ }^{8}$ We have shown in this work that structural changes of mitochondria, namely, matrix condensation and cristal unraveling, are a consequence of the decline in MMP. These structural changes result in the redistribution of cytochrome $c$ from the cristae to the intermembrane space, making it more susceptible to release.

The MMP can be decreased by several mechanisms including deficiency of oxidizable substrates for the mitochondria (Figure 1), blockage of respiration (Figure 2a and $3 \mathrm{c}$ ), or

\footnotetext{
Figure 6 Matrix configuration regulates the efficiency of cytochrome $c$ release and the susceptibility to apoptosis. (a) Mitochondria isolated from FL5.12 cells growing in the presence of IL-3 were incubated in MRB with $5 \mu \mathrm{M}$ rotenone and $7 \mathrm{mM}$ succinate with or without $50 \mu \mathrm{M}$ CCCP for $10 \mathrm{~min}$. Immuno-EM analysis using anticytochrome $c$ antibodies was performed. Three representative images of each treatment are shown. Arrows indicate cytochrome $c$ facing the intermembrane space in condensed mitochondria. (b) The number of immunodetected cytochrome $c$ particles was tallied from more than 50 mitochondria of each treatment. The percentage of cytochrome $c$ available to the intermembrane space is presented. (c) Isolated mitochondria were incubated with the indicated substrates for $10 \mathrm{~min}$ at room temperature followed by treatment with $5 \mu \mathrm{M}$ wild type or mutant $\mathrm{BH} 3$ peptide for $30 \mathrm{~min}$ at $37^{\circ} \mathrm{C}$. (i) Cytochrome $c$ release from these mitochondria was assessed using Western blot analysis. (ii) Quantification of cytochrome $c$ release of each wild-type $\mathrm{BH}$-treated sample by analyzing the different cytochrome $c$ proteins bands with Scion Image for Windows. The densities of equivalent areas from the mutant $\mathrm{BH}$-treated samples were deducted from the corresponding wild-type BH3-treated bands. (d) FL5.12 cells were incubated with or without $5 \mu \mathrm{M}$ rotenone or $10 \mu \mathrm{M} \mathrm{BH} 3 \mathrm{I}-2$ as indicated. Cell death was analyzed $12 \mathrm{~h}$ later by propidium iodide exclusion and assessed by FACS analysis. The results are the average and standard deviation of triplicate samples. cyt $\mathrm{c}=$ cytochrome $c$; wt $\mathrm{BH} 3$ and mut $\mathrm{BH} 3=$ wild type and mutant $\mathrm{BH} 3$ peptides respectively; $\mathrm{mal} / \mathrm{glut}=$ malate and glutamate
} 
uncoupling of the inner membrane (Figure $3 a$ and b). In each case, the consequence of the decline in MMP is condensation of the matrix. Still, the most compelling evidence for the direct relation between MMP and matrix configuration comes from the ability of an artificially created $\Delta \mathrm{pH}$ to convert mitochondria from a condensed to an orthodox configuration (Figure $5 b$ ). These results could also suggest that mitochondrial matrix swelling, observed in many pathological conditions, $7,14,21,22$ may be a consequence of cytosol acidification, a phenomenon frequently observed during necrotic cell death. ${ }^{23-27}$

The question remains as to what role does matrix configuration play in cells in which depolarization of the inner membrane is not observed prior to cytochrome $c$ release. ${ }^{28}$ It will be interesting to see whether mitochondrial structural changes, brought about by other mechanisms such as mitochondrial fission, ${ }^{29}$ or tBid-mediated matrix remodeling, ${ }^{8}$ will have a role in potential-independent mitochondria reorganization during apoptosis.

In addition to providing a link between the structure and function of mitochondria and apoptosis, our results may have implications for future administrations of cytochrome $c$ releasing drugs to induce apoptosis in vivo. $\mathrm{BH} 3$ peptides are potent cytochrome $c$ releasing agents, and molecules that can mimic $\mathrm{BH} 3$ peptides' structure and function are candidate drugs for treating tumors that overexpress $\mathrm{Bcl}-2$. In this setting, mitochondrial depolarizing drugs or other drugs that lead to matrix condensation may augment cytochrome $c$ release and facilitate apoptosis.

\section{Materials and Methods}

\section{Cell culture and analysis}

The murine IL-3-dependent cell lines FL5.12 were cultured at $37^{\circ} \mathrm{C}$ and $5 \% \mathrm{CO}_{2}$ in RPMI medium supplemented with $10 \%$ fetal bovine serum, penicillin/streptomycin, $10 \mathrm{mM}$ HEPES, $50 \mu \mathrm{M}$ 2-mercaptoethanol and $300 \mathrm{pg} / \mathrm{ml} \mathrm{IL}-3$. For apoptosis induction by IL-3 withdrawal, cells were washed 3 times in serum free RPMI medium and resuspended in complete medium with or without IL-3. For apoptosis induction by $\mathrm{BH} 3 \mathrm{I}-2$, cells were incubated in complete medium with IL-3 and treated for $12 \mathrm{~h}$ with either DMSO (control), $5 \mu \mathrm{M}$ rotenone, $10 \mu \mathrm{M} \mathrm{BH} 3 \mathrm{I}-2$, or rotenone and $\mathrm{BH} 3 \mathrm{I}-2$ together. Viability was determined by propidium iodide $(1 \mu \mathrm{g} / \mathrm{ml})$ exclusion and analyzed by FACS. To analyze MMP in cells, cells were treated as described in the text and incubated with $25 \mathrm{nM}$ TMRE for $30 \mathrm{~min}$ followed by FACS analysis.

\section{Mitochondrial isolation}

Mitochondria were isolated using the differential centrifugation method. Cells $\left(2.5 \times 10^{8}-5 \times 10^{8}\right)$ were harvested and placed on ice for $15 \mathrm{~min}$, centrifuged at $500 \times g$ for $5 \mathrm{~min}$ at $4^{\circ} \mathrm{C}$, washed with ice-cold PBS and subsequently washed with ice-cold mitochondrial isolation buffer (MIB) (200 mM mannitol, $70 \mathrm{mM}$ sucrose, $1 \mathrm{mM}$ EGTA, $10 \mathrm{mM} \mathrm{HEPES,} 0.5 \mathrm{mg} /$ $\mathrm{ml} \mathrm{BSA} ; \mathrm{pH}=7.4)$. Cells were resuspended in ice-cold MIB and then homogenized in a syringe-driven cell disruptor. The lysate was spun at $800 \times g$ for $10 \mathrm{~min}$ at $4^{\circ} \mathrm{C}$. Supernatants were removed and spun at $10000 \times g$ for $10 \mathrm{~min}$ at $4^{\circ} \mathrm{C}$. Pellets were resuspended in MIB and protein concentration was defined using the Bradford Reagent (Bio-Rad Laboratories).

\section{EM preparation}

Cells grown in the presence or absence of IL-3 were washed once with a fixative solution ( $2 \%$ glutaraldehyde in $0.1 \mathrm{M}$ sodium cacodilate; $\mathrm{pH}=7.4$ ) and spun down immediately. Fixative was then added to the pellets and samples were kept at $4^{\circ} \mathrm{C}$ overnight then embedded and sectioned $(70 \mathrm{~nm})$ in Epon following analysis by standard EM procedures. For mitochondria analysis, $200 \mu \mathrm{g}$ of mitochondria were added to $1 \mathrm{ml}$ of mitochondrial reaction buffer (MRB) (250 mM sucrose, $20 \mathrm{mM}$ HEPES, $10 \mathrm{mM} \mathrm{KCl}, 3 \mathrm{mM} \mathrm{KH}_{2} \mathrm{PO}_{4}, 1.5 \mathrm{mM} \mathrm{MgCl}_{2}, 1 \mathrm{mM} \mathrm{EGTA}, 0.5 \mathrm{mg} / \mathrm{ml} \mathrm{BSA}$; $\mathrm{pH}=7.4$ ) with or without $7 \mathrm{mM}$ succinate or $7 \mathrm{mM}$ (each) malate and glutamate. Samples were incubated for $10 \mathrm{~min}$ at $30^{\circ} \mathrm{C}$. Mitochondria were spun at $10000 \times \mathrm{g}$ for $10 \mathrm{~min}$ at $4^{\circ} \mathrm{C}$. Buffer was replaced with $1 \mathrm{ml}$ of fixative and samples were fixed overnight at $4^{\circ} \mathrm{C}$ and analyzed.

For immuno-EM analysis, following isolation and treatments, mitochondria were fixed in $2 \%$ paraformaldehyde, $0.1 \%$ gutaraldehde in $0.1 \mathrm{M}$ sodium cacodilate overnight, then dehydrated, embedded and sectioned $(90 \mathrm{~nm})$ in LR white. Sections were washed in Tris-buffered saline (TBS) and incubated in blocking buffer ( $1 \%$ ovalbumin, $0.2 \%$ cold water fish skin gelatin (Sigma, St. Louis, MO, USA) in PBS) for $1 \mathrm{~h}$ at $4^{\circ} \mathrm{C}$, washed again and incubated with rabbit polyclonal anticytochrome $c$ antibodies $(10 \mu \mathrm{g} /$ $\mathrm{ml}$ ) (Santa Cruz, CA, USA) or control serum, overnight at $4^{\circ} \mathrm{C}$. After washing with TBS, sections were incubated with 1:10 dilution of secondary antibodies (gold ( $5 \mathrm{~nm})$-conjugated goat anti-rabbit) (Sigma, St. Louis, MO, USA) for $1 \mathrm{~h}$ at room temperature, washed in PBS and analyzed by EM.

\section{MMP and matrix configuration analysis in vitro}

Isolated mitochondria $(500 \mu \mathrm{g})$ were diluted in $3 \mathrm{ml}$ of MRB at room temperature, in the presence or absence of $5 \mu \mathrm{M}$ rotenone. For membrane potential analysis, the rhodamine 123 quenching technique was used. ${ }^{13}$ Mitochondria were incubated with $0.3 \mu \mathrm{M}$ rhodamine 123 and analyzed in a spectrofluorometer using an excitation and emission wavelength of $500 \pm 2.5$ and $525 \pm 2.5 \mathrm{~nm}$, respectively. Baseline fluorescence was recorded for $300 \mathrm{~s}$ followed by the sequential addition of oxidizable substrates (7 mM succinate or $7 \mathrm{mM}$ (each) malate and glutamate) and $50 \mu \mathrm{M}$ CCCP as indicated in the text. For matrix configuration analysis $90^{\circ}$ light scattering technique was used. ${ }^{13}$ Mitochondria were placed in the spectrofluorometer and light diffraction was detected by setting both the excitation and the emission wavelength to $520 \pm 2 \mathrm{~nm}$. To study the effects of volume changes on rhodamine 123 fluorescence in the absence of changes in MMP, mitochondria were depolarized with $50 \mu \mathrm{M} \mathrm{CCCP} \mathrm{and}$ $50 \mathrm{nM}$ valinomycin, incubated in $100 \mathrm{mM}$ sucrose buffer ( $100 \mathrm{mM}$ sucrose, $20 \mathrm{mM}$ HEPES, $1 \mathrm{mM}$ EGTA, $0.5 \mathrm{mg} / \mathrm{ml} \mathrm{BSA}$; $\mathrm{pH}=7.4$ ) and $90^{\circ}$ light scattering or rhodamine 123 quenching technique were used. Sucrose concentration was increased every $200 \mathrm{~s}$ by $50 \mathrm{mM}$ to follow changes in matrix volume and rhodamine 123 fluorescence.

\section{Cytochrome $c$ release analysis}

Isolated mitochondria $(100 \mu \mathrm{g})$ were placed in $100 \mu \mathrm{l}$ of MRB for $8 \mathrm{~min}$ at room temperature with $7 \mathrm{mM}$ succinate or $7 \mathrm{mM}$ (each) malate and glutamate. Where indicated (in Figure $6 \mathrm{c}$ ) $5 \mu \mathrm{M}$ rotenone or $50 \mu \mathrm{M} \mathrm{CCCP}$ were added. After 2 more minutes incubation at room temperature, $5 \mu \mathrm{M}$ wild-type (wt) BH3 peptide derived from human Bid (amino acids 80-99) or $5 \mu \mathrm{M}$ mutant (mut) BH3 peptide with two point mutations (L90A and D95A) were added and mitochondria were incubated for $30 \mathrm{~min}$ at $37^{\circ} \mathrm{C}$. Then, mitochondria were spun down $\left(10000 \times g, 4^{\circ} \mathrm{C}, 10 \mathrm{~min}\right)$ and the supernatant was collected and used for Western blot analysis using anticytochrome $c$ antibody clone $7 \mathrm{H} 8.2 \mathrm{C} 12$ (BD Pharmingen). 


\section{Acknowledgements}

We thank the Biomedical Imaging Core Facility at the University of Pennsylvania for excellent performance with EM analysis and Ayala King and Mary Selak for fruitful discussions and editorial advice. $\mathrm{BH} 3$ peptides were a gift of Kevin Tomaselli of Idun Pharmaceuticals. Eyal Gottlieb is supported by a special fellowship from the Leukemia and Lymphoma Society.

\section{References}

1. Gottlieb RA (2000) Mitochondria: execution central. FEBS Lett. 482: 6-12

2. Wang $X$ (2001) The expanding role of mitochondria in apoptosis. Genes Dev. 15: 2922-2933

3. Hengartner MO (2000) The biochemistry of apoptosis. Nature 407: 770-776

4. Adrain C and Martin SJ (2001) The mitochondrial apoptosome: a killer unleashed by the cytochrome seas. Trends Biochem. Sci. 26: 390-397

5. Vander Heiden MG, Plas DR, Rathmell JC, Fox CJ, Harris MH and Thompson $\mathrm{CB}$ (2001) Growth factors can influence cell growth and survival through effects on glucose metabolism. Mol. Cell. Biol. 21: 5899-5912

6. Kan O, Baldwin SA and Whetton AD (1994) Apoptosis is regulated by the rate of glucose transport in an interleukin 3 dependent cell line. J. Exp. Med. 180: 917-923

7. Bernardi P, Petronilli V, Di Lisa F and Forte M (2001) A mitochondria perspective on cell death. Trends Biochem. Sci. 26: 112-117

8. Scorrano L, Ashiya M, Buttle K, Weiler S, Oakes SA, Mannella CA and Korsmeyer SJ (2002) A distinct pathway remodels mitochondrial cristae and mobilizes cytochrome $c$ during Apoptosis. Dev. Cell. 2: 55-67

9. Vander Heiden MG, Chandel NS, Williamson EK, Schumacker PT and Thompson CB (1997) Bcl-xL regulates the membrane potential and volume homeostasis of mitochondria. Cell 91: 627-637

10. Scarlett JL, Sheard PW, Hughes G, Ledgerwood EC, Ku HH and Murphy MP (2000) Changes in mitochondrial membrane potential during staurosporineinduced apoptosis in Jurkat cells. FEBS Lett. 475: 267-272

11. Khaled AR, Reynolds DA, Young HA, Thompson CB, Muegge K and Durum SK (2001) Interleukin-3 withdrawal induces an early increase in mitochondrial membrane potential unrelated to the $\mathrm{Bcl}-2$ family. Roles of intracellular $\mathrm{pH}$, ADP transport, and F(0)F(1)-ATPase. J. Biol. Chem. 276: 6453-6462

12. Hackenbrock CR (1966) Ultrastructural bases for metabolically linked mechanical activity in mitochondria. J. Cell Biol. 30: 269-297

13. Zamzami N, Maisse C, Metivier D and Kroemer G (2001). Measurement of membrane permeability and permeability transition of mitochondria. In Methods in Cell Biology, Pon LA and Schon EA (eds) (San Diego: Academic Press) pp. $147-158$
14. Halestrap AP (1989) The regulation of the matrix volume of mammalian mitochondria in vivo and in vitro and its role in the control of mitochondrial metabolism. Biochim. Biophys. Acta 973: 355-382

15. Frey $T G$ and Mannella CA (2000) The internal structure of mitochondria. Trends Biochem. Sci. 25: 319-324

16. Degterev A, Lugovskoy A, Cardone M, Mulley B, Wagner G, Mitchison T and Yuan J (2001) Identification of small-molecule inhibitors of interaction between the BH3 domain and Bcl-xL. Nat. Cell. Biol. 3: 173-182

17. Zamzami N, Marchetti P, Castedo M, Decaudin D, Macho A, Hirsch T, Susin SA, Petit PX, Mignotte B and Kroemer G (1995) Sequential reduction of mitochondrial transmembrane potential and generation of reactive oxygen species in early programmed cell death. J. Exp. Med. 182: 367-377

18. Gottlieb E, Vander Heiden MG and Thompson CB (2000) Bcl-XL prevents the initial decrease in mitochondrial membrane potential and subsequent reactive oxygen species production during tumor necrosis factor alpha-induced apoptosis. Mol. Cell. Biol. 20: 5680-5689

19. Gottlieb E, Armour SM and Thompson CB (2002) Mitochondrial respiratory control is lost during growth factor deprivation. Proc. Natl. Acad. Sci. USA 99: 12801-12806

20. Goldstein JC, Waterhouse NJ, Juin P, Evan Gl and Green DR (2000) The coordinate release of cytochrome $c$ during apoptosis is rapid, complete and kinetically invariant. Nat. Cell. Biol. 2: 156-162

21. Kroemer G, Dallaporta B and Resche-Rigon M (1998) The mitochondrial death/ life regulator in apoptosis and necrosis. Annu. Rev. Physiol. 60: 619-642

22. Kroemer G and Reed JC (2000) Mitochondrial control of cell death. Nat. Med. 6 : 513-519.

23. Kalimo H, Rehncrona S, Soderfeldt B, Olsson Y and Siesjo BK (1981) Brain lactic acidosis and ischemic cell damage: 2. Histopathology. J. Cereb. Blood Flow Metab. 1: 313-327

24. Ding D, Moskowitz SI, Li R, Lee SB, Esteban M, Tomaselli K, Chan J and Bergold PJ (2000) Acidosis induces necrosis and apoptosis of cultured hippocampal neurons. Exp. Neurol. 162: 1-12

25. Aki T, Mizukami Y, Oka Y, Yamaguchi K, Uemura K, Fujimiya T and Yoshida K (2001) Phosphoinositide 3-kinase accelerates necrotic cell death during hypoxia. Biochem. J. 358: 481-487

26. Takahashi K, Ohyabu Y, Schaffer SW and Azuma J (2001) Cellular characterization of an in-vitro cell culture model of seal-induced cardiac ischaemia. J. Pharm. Pharmacol. 53: 379-386

27. Affar el B, Shah RG, Dallaire AK, Castonguay V and Shah GM (2002) Role of poly(ADP-ribose) polymerase in rapid intracellular acidification induced by alkylating DNA damage. Proc. Natl. Acad. Sci. USA 99: 245-250

28. Waterhouse NJ, Goldstein JC, von Ahsen O, Schuler M, Newmeyer DD and Green DR (2001) Cytochrome $c$ maintains mitochondrial transmembrane potential and ATP generation after outer mitochondrial membrane permeabilization during the apoptotic process. J. Cell Biol. 153: 319-328

29. Frank S, Gaume B, Bergmann-Leitner ES, Leitner WW, Robert EG, Catez F, Smith CL and Youle RJ (2001) The role of dynamin-related protein 1, a mediator of mitochondrial fission, in apoptosis. Dev Cell. 1: 515-525 